J. Clin. Chem. Clin. Biochem.

Vol. 20, 1982, pp. 861-873

\title{
Technik der infrarotspektroskopischen Harnsteinanalyse
}

Von $A$. Hesse

Harnsteinforschungsstelle der Urologischen Universitätsklinik Bonn (Direktor: Prof. Dr. W. Vahlensieck) und

\section{K. Molt}

Bodenseewerk Perkin-Elmer \& Co. GmbH

(Eingegangen am 6. Juli/1. Oktober 1982)

Zusammenfassung: Für die qualitative und quantitative Analyse von Harnsteinen mit Hilfe der Infrarotspektroskopie wird die Technik der Durchführung erprobt und erläutert. Es wurden Infrarotspektren für alle bekannten, in Harnsteinen auftretenden Substanzen aufgenommen und interpretiert. An Beispielen wurden die Methoden der qualitativen und quantitativen Auswertung geprüft. Durch Anwendung einer IR-Datenstation wurden die Grundlagen für eine rechnerinterstützte automatische Auswertung von Infrarotspektren zur Harnsteinanalyse geschaffen.

\section{The technique of analysis of urinary calculi by infra-red spectroscopy}

The technical procedure is tested and elucidated for the qualitative and quantitative analysis of urinary calculi by infrared spectroscopy. Infra-red spectra of all substances, known to occur in urinary calculi were recorded and interpreted. The methods of the qualitative and quantitative evaluation were tested on various examples. Using an infra-red data station, the basis of a computerized automatic evaluation of infra-red spectra for the analysis of urinary calculi was established.

\section{Einführung}

Für eine gezielte Metaphylaxe bei Harnsteinleiden ist die Steinanalyse einer der wichtigstèn klinischchemischen Befunde. Dabei ist es nicht ausreichend, aus einer Lösung des Harnsteines die vorhandenen İonen zu bestimmen, zumal Ringversuche zur Qualitätskontrolle der Harnsteinanạalyse einè hohe Fehlerquote bei Anwendung vón chemischen Methoden ergaben (1). Heute ist es notwendig, aus verschiedenen Zonen des Ḧarnsteines den unveränderten Kristalloder Molekülzustand der Phaseñ zu ermitteln. Daraus können differenżierte Aussagen zur Genese und der möglichen Therapie gewonnen werden (2).

Dafür sind Methoden geeignet, die mit geringem Probenmaterial $(1-10 \mathrm{mg})$ auskommen und eine Direktuntersuchung des Mätèrials ermöglichen. Mit der Röntgendiffraktion (3) können alle gut kristạllisierten. Bestandteile identifiziert werden. Dagegen erlaubt die Infrarotspektroskopie die direkte Identifizierung von Atomgruppen mit kovalenten Bedingungen in Harnsteinen $(4,5)$. In der vorliegenden Arbeit werden die Technik der infrarotspektroskopischen Harnsteinanaalyse mitgeteilt und für alle bekannten Harnsteinsubstanzen die Infrarotspektren interpretiert sowie die qualitativen und quantitativen Methoden der Harnsteinanalyse erläutert.

\section{Material und Methode}

Aus synthetischen Materialien und Harnsteinsubstanzen wurden Infrarotspektren mit der Kaliumbromid-Preßtechnik hergestellt.

\footnotetext{
Vorbereitung der Probe für die Messung .

Unabhängig von der Art der Harnsteinanalyse ist ein richtiges Ergebnis nur zu erhalten, wenn eine repräsentative Probennahme erfolgt. Auch kleine Steine können aus mehreren Komponenten bestehen und untergeordnete Mischanteile für die Auslösung der Steinbildung verantwortlich sein. Deshalb wurde bei uneinheitlicher makroskopischer Struktur eine Analyse aus mehreren Zonen des Harnsteines angefertigt. Könnte dies bei kleineren Steinen nicht erfolgen, wurde die gesamte Probe in einem Achatmörser zermahlen (Tab. 1).
} 
Tab. 1. Aufwand für eine infrarotspektroskopische Harnsteinanalyse.

\begin{tabular}{|c|c|c|c|}
\hline 1. Beurteilung des Harnsteines & \multicolumn{2}{|c|}{$\begin{array}{l}\text { - Gewicht } \\
\text { - Oberflächenstruktur } \\
\text { - Farbe }\end{array}$} & \multirow[b]{2}{*}{$\begin{array}{l}\text { Durchmesser } \\
\text { zersägen und aus verschiedenen Zonen repräsentative } \\
\text { Proben nehmen } \\
\text { Durchmesser } \\
\text { insgesamt im Achatmörser zermahlen }\end{array}$} \\
\hline 2. Probengewinnung & - Steine $<3$ & $\mathrm{~mm}$ & \\
\hline 3. Probenmenge & $\begin{array}{l}-0,5-1,5 \\
-10-20\end{array}$ & mg & $\begin{array}{l}\text { Normalpreßwerkzeug } \\
\text { (Durchmesser des Preßlings } 13 \mathrm{~mm} \text { ) } \\
\text { Mikropreßwerkzeug } \\
\text { (Durchmesser des Preßlings } 1,5 \mathrm{~mm} \text { ) }\end{array}$ \\
\hline $\begin{array}{l}\text { 4. Trägermaterial } \\
\mathrm{KBr} \text { optisch rein }\end{array}$ & $\begin{array}{l}-250-300 \\
-\quad 5-7\end{array}$ & $\begin{array}{l}\mathrm{mg} \\
\mathrm{mg}\end{array}$ & $\begin{array}{l}\text { Normalpreßwerkzeug } \\
\text { Mikropreßwerkzeug }\end{array}$ \\
\hline 5. Vermischen von $\mathrm{KBr}+$ Probe & $-20-30$ & s & $\begin{array}{l}\text { in Mahlkapsel ạus Achat oder Edèlstahl in Schwing- } \\
\text { mühle vermischen }\end{array}$ \\
\hline 6. Herstellen der Preßlinge & \multicolumn{2}{|c|}{ - unter Vakuum } & $\begin{array}{l}\text { (Ausschluß von Luftfeuchtigkeit) wird bei } 8 \mathrm{t} / \mathrm{cm}^{2} \text { der } \\
\text { Preßling gepreßt }\end{array}$ \\
\hline 7. Gesamtzeit für Probenvorbereitung 1.-6. & \multicolumn{2}{|l|}{$-5 \min$} & \\
\hline 8. Aufnahme des IR-Spektrums & \multicolumn{2}{|c|}{ - Analysenżeit $8 \mathrm{~min}$} & $\cdot$ \\
\hline 9. Auswertung & \multicolumn{3}{|c|}{ - in Abhängigkeit von Erfahrung und Methode 2-10 min } \\
\hline 10. Gesamtzeit & \multicolumn{3}{|c|}{$-15-20 \mathrm{~min}$, effektive Arbeitszeit 7-15 $\mathrm{min}$} \\
\hline 11. Kosten an Verbrauchsmaterial & \multicolumn{3}{|c|}{ - Strom, 0,05 DM für KBr, 0,60 DM für Sçhreiberpapier } \\
\hline
\end{tabular}

Für die infrarotspektroskopische Untersuchung muß das Harnsteinmaterial mit einer inerten Trägersubstanz, die in dem zu untersuchenden Spektralbereich keine Absorptionen zeigt, homogenisiert werden. Dázu eignet sich am besten optisch reines Kaliumbromid. Das Harnsteinmaterial wurde mit der Trägersubstanz in einer Schwingmühle zermahlen. Für eine reproduzierbare Auswertung mit Geräten ohne Datenverarbeitung müssen beide Komponenten vor der Vermischung abgewogen werden. Näh dem Mahlprozeß wurde aus dem Gemisch in einer Preßvorrichtung ein glasklarer Preßling hergestellt. Dieser Preßling wurde dann in den Strahlengang des IR-Spektrometers gebracht und das Spektrum aufgezeichnet.

In Tabelle 1 ist der Aufwand für eine Harnsteinanalyse mit den technischen Daten zusammengefaßt.

\section{Aufnahme des Infrarot-Spektrums}

Bei konstanten Bedingungen, die für die Untersuchung von Kaliumbromid-Preßlingen abhängig vom Gerät leicht eingestellt werden können, wurde das IR-Spektrum aufgenommen. Dabei ist es für Harnsteinsubstanzen notwendig, ein Gerät zu verwenden, das im Wellenzahlbereich von $4000 \mathrm{~cm}^{-1}$ bis mindestens $400 \mathrm{~cm}^{-1}$ messen kann.

\section{Ergebnisse}

\section{Qualitative Analyse}

Für die qualitative Analyse wurden Vergleichsspektren von Reinsubstanzen für alle in Harnsteinen auftretenden Verbindungen genommen. In den Tafeln $\mathrm{I}-\mathrm{VI}$ sind die IR-Spektren für alle wichtigen Harnsteinsubstanzen zusammengestellt. Diese Vorlagen können direkt für eine Auswertung verwendet werden. Zum besseren Verstänḍis werden im folgenden die Spektren erläutert.

\section{Harnsäure}

Die IR-Spektren von Harnșäure und ihren Salzen sind sehr bandenreich. Starke Absorptionen sind auch bei Gemischen gut zuzuordnen. Die Bandengruppierüng von $400-800 \mathrm{~cm}^{-1}$ ist besonders wichtig; in diesem Bereich liegen die meisten Ünterschiede zu den anderen im Harnstëin vorkommenden Purịnkörpern. Andere charakteristische Absorptionen liegén bei 1591, 1675, 2825 und $3010 \mathrm{~cm}^{-1}$.

\section{Harnsäure-Dihydrat}

Zur Unterscheidung vön wasserfreier Harnșäure ist besonders auf die Veränderungen an der Bande bei $515 \mathrm{~cm}^{-1} \mathrm{zu}$ achten. Die Bande wird breiter und erhält eine Schulter. Des weiteren fehlt gegenüber der Harnsäure die Bande bei $873 \mathrm{~cm}^{-1}$, und die Absorptionen bei $1303,1346,1403$ und $1430 \mathrm{~cm}^{-1}$ haben eine andere Intensität und Form. Die Hydratwasserr-Bande bei . $3505 \mathrm{~cm}^{-1}$ ist ein wichtiges Indiz.

\section{Mono-ammoniumurat}

Die Bande bei $598 \mathrm{~cm}^{-1}$ ist eine eindeutige Abweichung zur Harnsäure und zu Harnsäure-Bihydrat. Außerdem ist die Bandengruppierung zwischen $1200-1600 \mathrm{~cm}^{-1}$ charakteristisch, in diesem Bereich sind auch wichtige Unterschiede zum Mono-natriumurat-Monohydrat. 


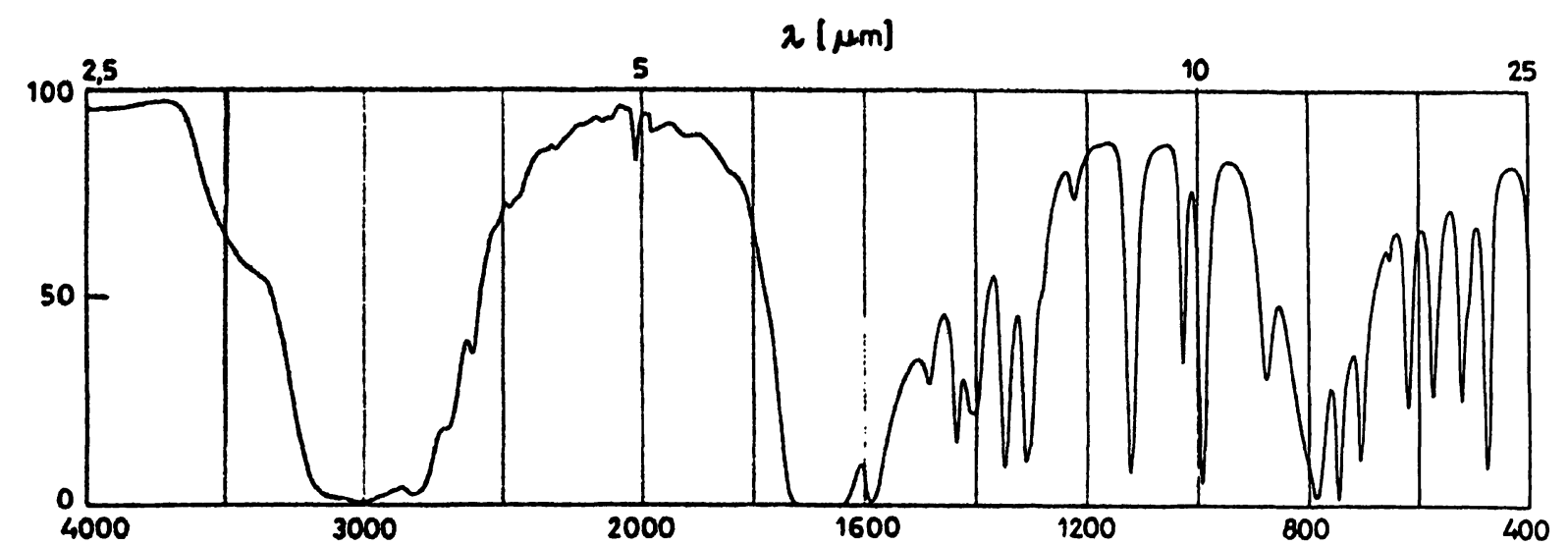

1 Harnsäure

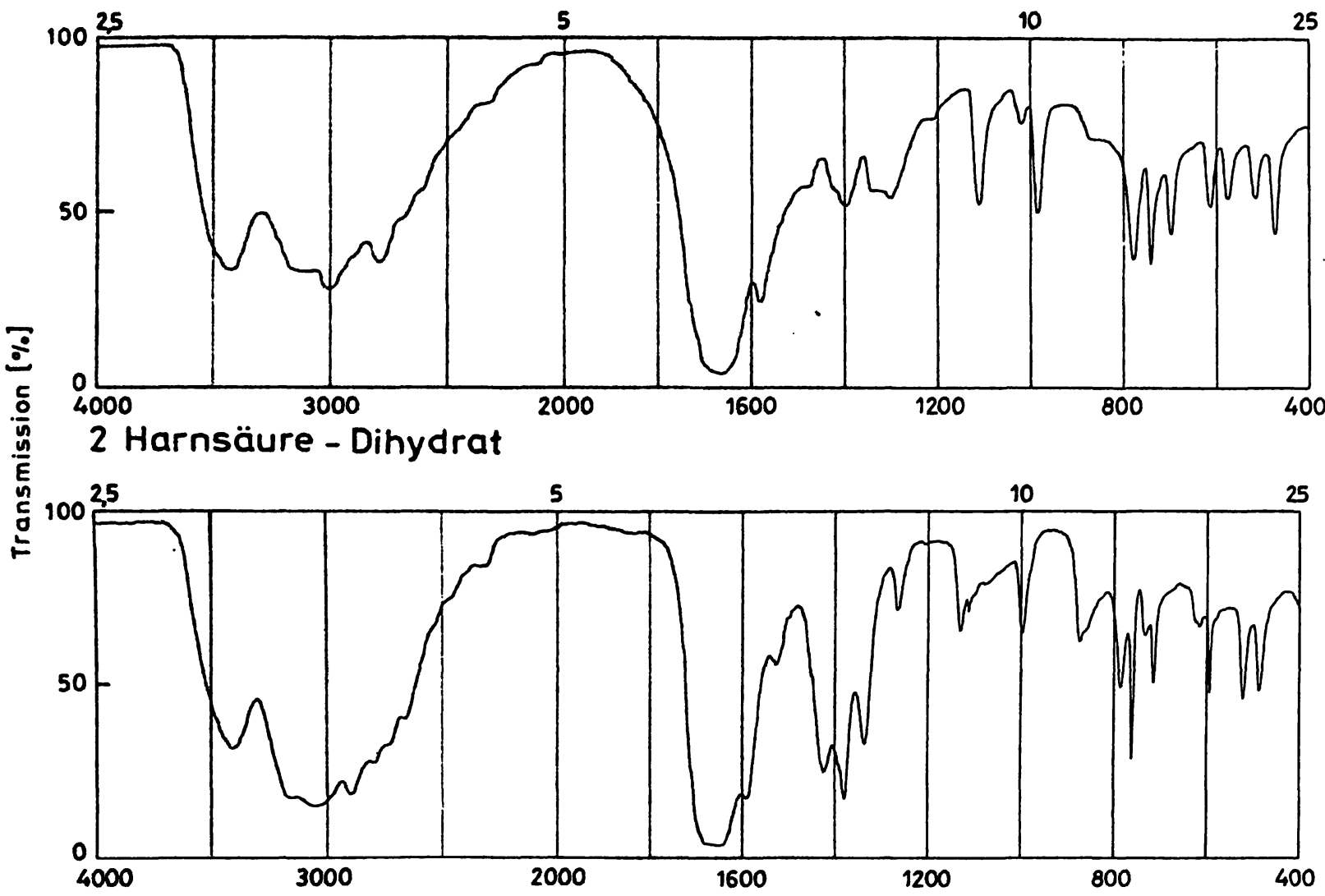

3 Mono-Ammonium-urat

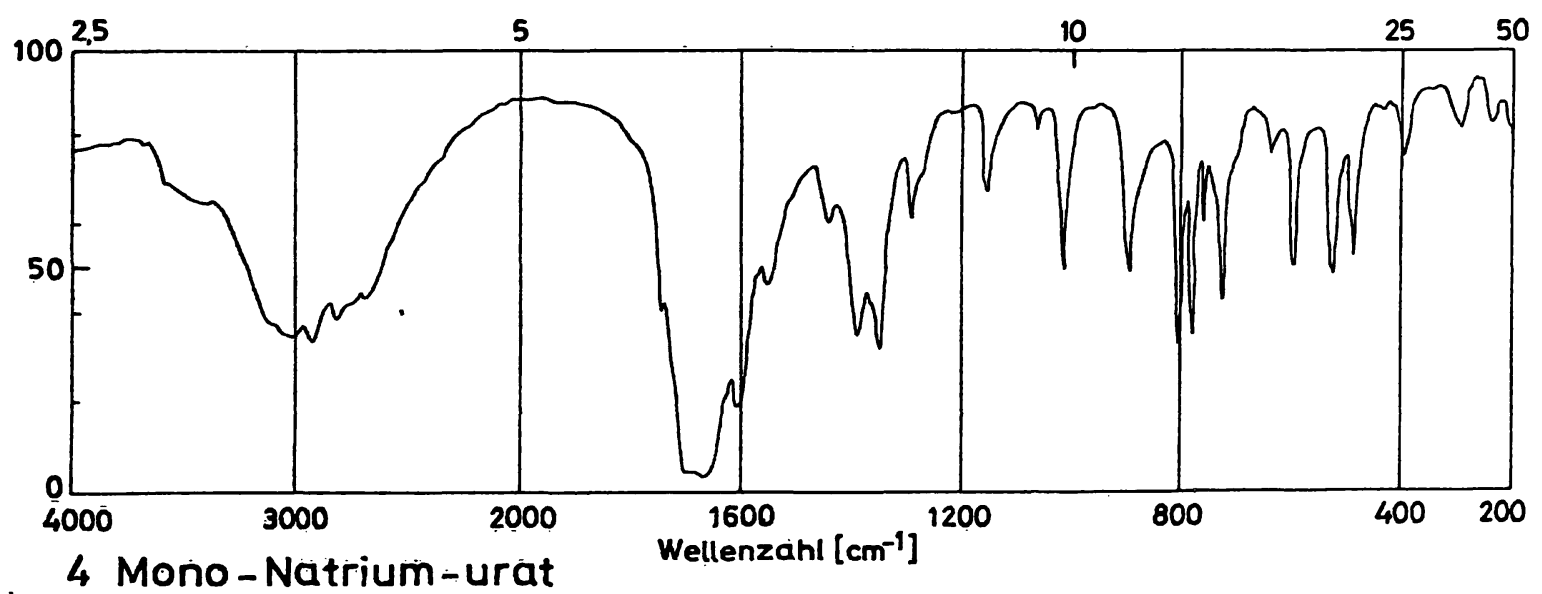

Tafel I Infrarotspektren voọ

1 Harnsäure

2 Harnsäure-Dihydrat
3 Mono-ammonium-urat

4 Mono-natrium-urat (beachte den Maßstab!)

Spektren reiner Substanzen als Vergleichs-Standards zur Harnsteinanalyse 
$\lambda[\mu \mathrm{m}]$
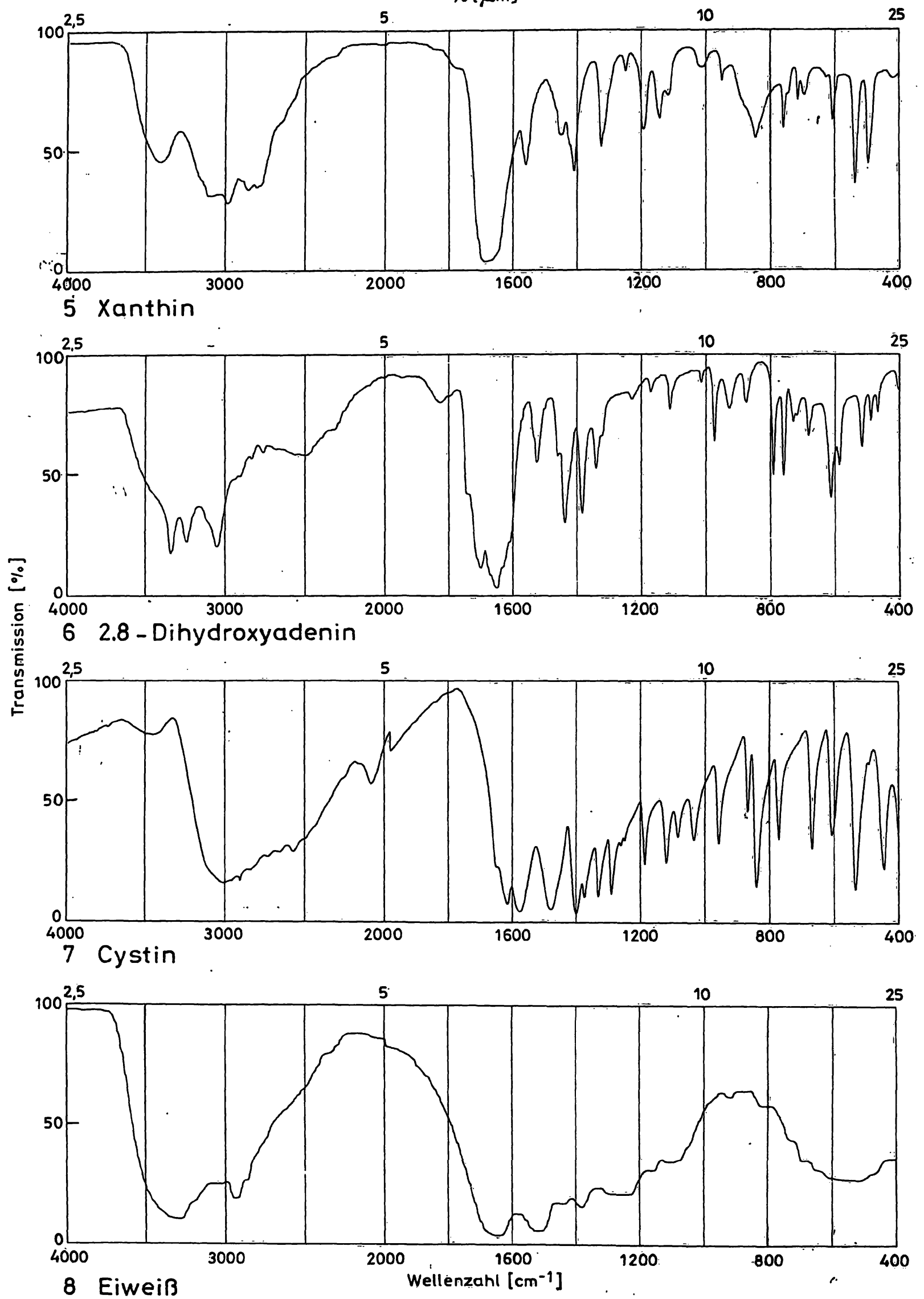

Tafel II Infrarotspektren von 


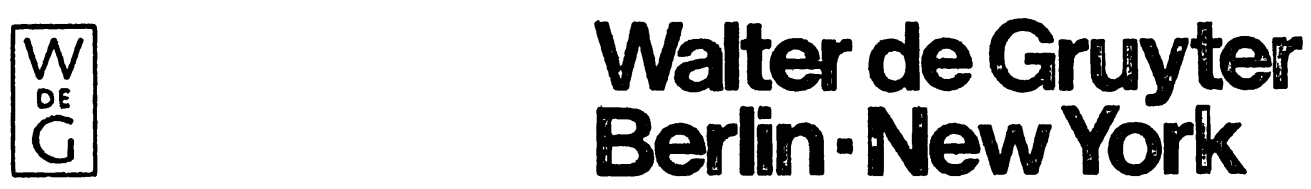

\section{Brandenburg} A. Wollmer (Editors)

\author{
K. Keck \\ P. Erb \\ (Editors)
}

\section{Insulin \\ Chemistry, Structure and Function of Insulin and Related Hormones \\ Proceedings of the Second International Insulin Symposium, Aachen, Germany, September 4-7, 1979}

1980. $17 \mathrm{~cm} \times 24 \mathrm{~cm} .752$ pages. Numerous figures.

Hardcover. DM 170,-; approx. US $\$ 81.00$ ISBN 3110081563

These proceedings of the Insulin Symposium present the current state of knowledge and research in the field of Insulin. The problems are presented and discussed from various standpoints (chemistry, biochemistry, biology, crystallography, immunology and medicine).

Contents: Introduction

Sections $|-X|$

Structure of Insulin - Peptide Synthesis - Semisynthesis and Chemical Modification - Radioactive Labelling and Separation Techniques - Receptors and Hormone-Receptor Interaction . Photo-Induced Hormone-Receptor Coupling - Structure, Binding, Activity - Degradation - Immunology - Biosynthesis, Storage, Evolution - Insulin-Related Hormones.

Abbreviations - Subject Index · Author Index.

\section{Basic and Clinical Aspects of Immunity to linsulin \\ Proceedings. International Workshop, September 28-October 1, 1980, Konstanz, Germany}

1981. $17 \mathrm{~cm} \times 24 \mathrm{~cm}$. XIV, 442 pages. Numerous illustrations. Hardcover. DM 140,-; approx. US $\$ 66.75$ ISBN 3110084406

In order to integrate the large quantities of information available regarding the chemistry and immunology of insulin as well as the clinical aspects of diabetes and its control, it is necessary to establish interdisciplinary lines of communication between specialists in these various fields. It was the intention of a Symposium held in Konstanz, FRG, from September 28 to October 1,1980, to bring together clinicians, immunologists and chemists active in insulin research, to provide an open forum to exchange ideas and experience, to establish contacts and to intensify cooperation between these groups. 


\section{Walter de Gruyter Berlin-New York}

F. Lottspeich A. Henschen K.-P. Hupe (Editors)

I. Molnar (Editor)

\section{A. Henschen \\ H. Graeff \\ F. Lottspeich (Editors)}

\section{Allen/Bienvenu/} Laurent/Suskind (Editors)
High Performance

Liquid Chromatography in Protein and Peptide Chemistry

Proceedings. International Symposium, January 1981, Max-Planck-Institute for Biochemistry, Martinsried/Munich, Germany

$1982.17 \mathrm{~cm} \times 24 \mathrm{~cm}$. XVIII, 388 pages. Numerous illustrations. Hardcover. DM 145,-; approx. US $\$ 69.25$ ISBN 3110085429

Chromatography is one of the most versatile methods in modern biochemical and pharmacological work. The field is rapidly developing and the book is intended to be an up-to-date survey of the most recent developments of methods related to protein, peptide and amino acid analysis.

Practical Aspects of Modern HPLC

Symposium, December 7-8, 1981, Berlin 1982. $17 \mathrm{~cm} \times 24 \mathrm{~cm}$. VIII, 449 pages. Numerous illustrations. Hardcover. DM 170,-; approx. US $\$ 81.00$ ISBN 3110088924

\section{Fibrinogen}

Recent Biochemical and Medical Aspects Proceedings. International Symposium, February 17-18, 1981, Max-Planck-Institute for Biochemistry Martinsried/Munich, Germany

$1982.17 \mathrm{~cm} \times 24 \mathrm{~cm} . \mathrm{X}, 400$ pages. Numerous illustrations. Hardcover. DM 135,-; approx. US \$64.50 ISBN 3110085437

\section{Marker Proteins in Inflammation} Proceedings of the Symposium Lyon, France, April 22-25, 1981

$1982.17 \mathrm{~cm} \times 24 \mathrm{~cm}$. XVI, 608 pages. Numerous illustrations. Hardcover. DM 185,-; approx. US \$88.25 ISBN 3110086255 


\section{Mono-natriumurat-Monohydrat}

Natriumurat kommt außerordentlich selten in Harnsteinen vor. Wichtig ist die Unterscheidung von Ammoniumurat; diese ist durch die Banden bei 719, 198, 1338,1380 und $1458 \mathrm{~cm}^{-1}$ gegeben.

\section{Xanthin}

Xanthin ist als Harnstein eine Rarität. Als Purin-Derivat besitzt es ein sehr bandenreiches Spektrum. Die Unterscheidung von anderen Purinkörpern ist unproblematisch. Typische Absorptionen liegen bei 497, 537, 613, $849,1120,1151,1200$ und $1697 \mathrm{~cm}^{-1}$.

\section{2.8-Dihydroxy-adenin}

Die Bandenfolge zwischen $450-1000 \mathrm{~cm}^{-1}$ unterscheidet sich typisch von anderen Purinkörpern. Charakteristisch sind die Absorptionen bei 3045,3238 und $3338 \mathrm{~cm}^{-1}$, die in keinem anderen Harnsteinmaterial auftreten.

\section{Cystin}

Charakteristische Banden für die -S-S- Banden liegen bei $400-500 \mathrm{~cm}^{-1}$. Die Bandengruppierung zwischen $1200-1600 \mathrm{~cm}^{-1}$ ist für die Identifizierung in Gemischen wichtig.

\section{Eiweiß}

Proteine haben eine typische, assoziierte NH-Valenzschwingungsbande bei $3300 \mathrm{~cm}^{-1}$. Die Amidbanden I und II bei 1650 und $1525 \mathrm{~cm}^{-1}$ sind ebenfalls charakteristisch. Die CH-Absorptionen bei $2880 \mathrm{~cm}^{-1}$ und $2970 \mathrm{~cm}^{-1}$ geben häufig den Hinweis für das Vorliegen von Eiweißen in Mischsteinen.

\section{Whewellit}

Die Unterscheidung der beiden Calciumoxalat-Hydrate Whewellit und Weddellit ist anhand der IR-Spektren sehr gut möglich. Für Whewellit liegen die charakakteristischen Absorptionen bei $655,781,883$ und $948 \mathrm{~cm}^{-1}$, und zum Unterschied zum Weddellit ist die Hydratwasser-Valenżschwingungsbande in 5 Einzelbanden von $3058-3488 \mathrm{~cm}^{-1}$ aufgespalten.

\section{Wéddellit}

Im Gegensatz zu Whewellit ist die Hydratwasserbande bei $3488 \mathrm{~cm}^{-1}$ breit und nicht aufgespalten. Die $\mathrm{C}=0$ Valenzschwingungsbande liegt bei $1640 \mathrm{~cm}^{-1}$. Charakteristisch zur Unterscheidung von Whewellit sind auch die Absorptionen bei 608 und $910 \mathrm{~cm}^{-1}$.

\section{Whitlockit, Apatit}

Beide Verbindungen sind chemisch Tricalciumphosphate und deshalb im IR-Spektrum identisch. Das Spektrum ist sehr bandenarm. Die Hauptabsorptionen liegen bei 1032,562 und $602 \mathrm{~cm}^{-1}$.

\section{Hydroxylapatit}

Zur Unterscheidung vom Apatit ist die Bande bei 631 $\mathrm{cm}^{-1}$ charakteristisch. Bei Untersuchung des Bereiches von $200-400 \mathrm{~cm}^{-1}$ werden typische Banden nachgewiesen. In Harnsteinen und anderem biologischen Material konnte mit der IR-Spektroskopie ein solches Phosphat nicht nachgewiesen werden.

\section{Carbonatapatit}

Die Carbonatbanden bei 850,1414 und $1457 \mathrm{~cm}^{-1}$ sind für dieses Phosphat charakteristisch und geben eine leichte Unterscheidungsmöglichkeit zum Apatit. Das Carbonation substituiert im Ca-Phosphat eine Phosphatgruppe. Es liegt nicht als $\mathrm{CaCO}_{3}$ vor.

\section{Amorphes Calciumphosphat}

Calciumphosphat kann sehr schlecht kristallisiert im Harnstein und auch anderen Biomineralisaten auftreten. Die Aufspaltung der Bande bei 562 und $602 \mathrm{~cm}^{-1}$ (siehe Apatit) ist ein Maß für den Kristallisationsgrad. Im amorphen Ca-Phosphat findet man eine breite Bande bei $575 \mathrm{~cm}^{-1}$.

\section{$15 \mathrm{MgNH}_{4} \mathrm{PO}_{4} \times 1 \mathrm{H}_{2} \mathrm{O}$}

Dieses Magnesiumphosphat ist eine sehr seltene Harnsteinkomponente; es muß als Alterungsprodukt von Struvit angesehen werden. Die Lage der Banden zwischen $400-800 \mathrm{~cm}^{-1}$ ist von den Calciumphosphaten deutlich abweichend und bei $1655 \mathrm{~cm}^{-1}$ tritt eine charakteristische Bande für das Kristallwasser im Molekïl auf.

\section{Struvit}

Struvit wird vor allem bei gleichzeitig bestehender Infektion in Steinen gefunden und kann durch die typischen Banden bei 566,750 und $990 \mathrm{~cm}^{-1}$ auch in Gemischen leicht identifiziert werden.

\section{Brushit}

Durch die mehrfach aufgespaltene Phosphatbande zwischen 800-1200 $\mathrm{cm}^{-1}$ kann dieses saure Calciumphosphat gut von anderen Phosphaten unterschieden werden. 


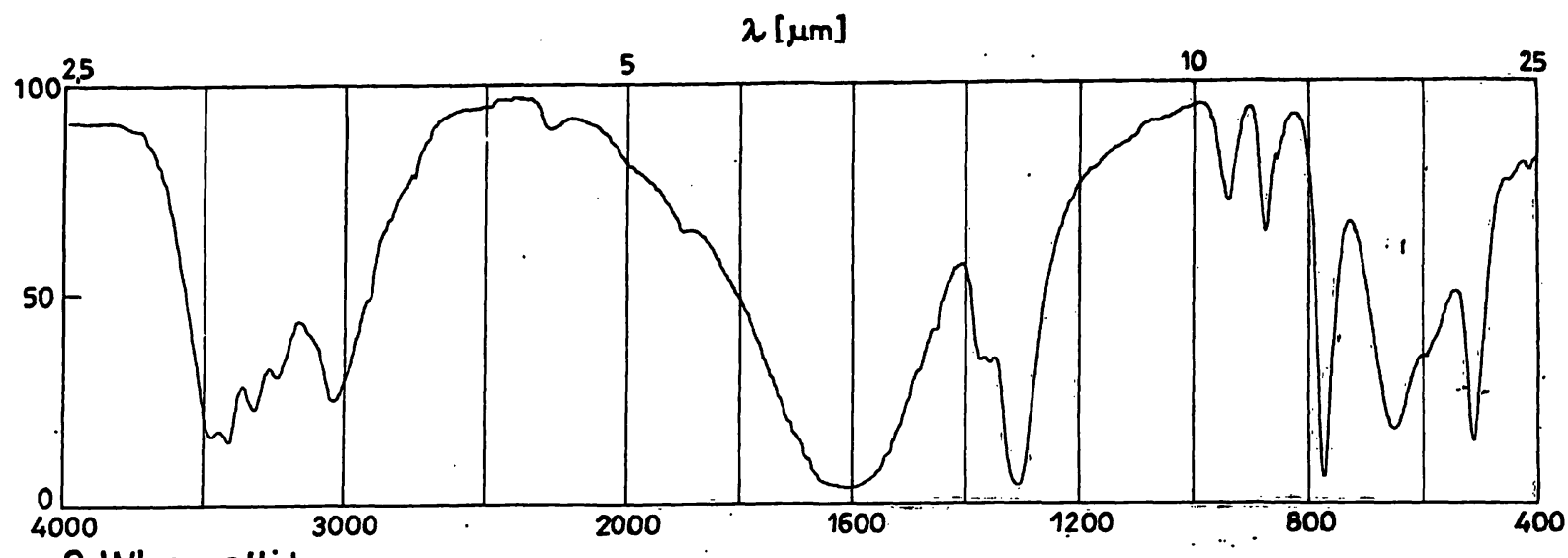

9 Whewellit

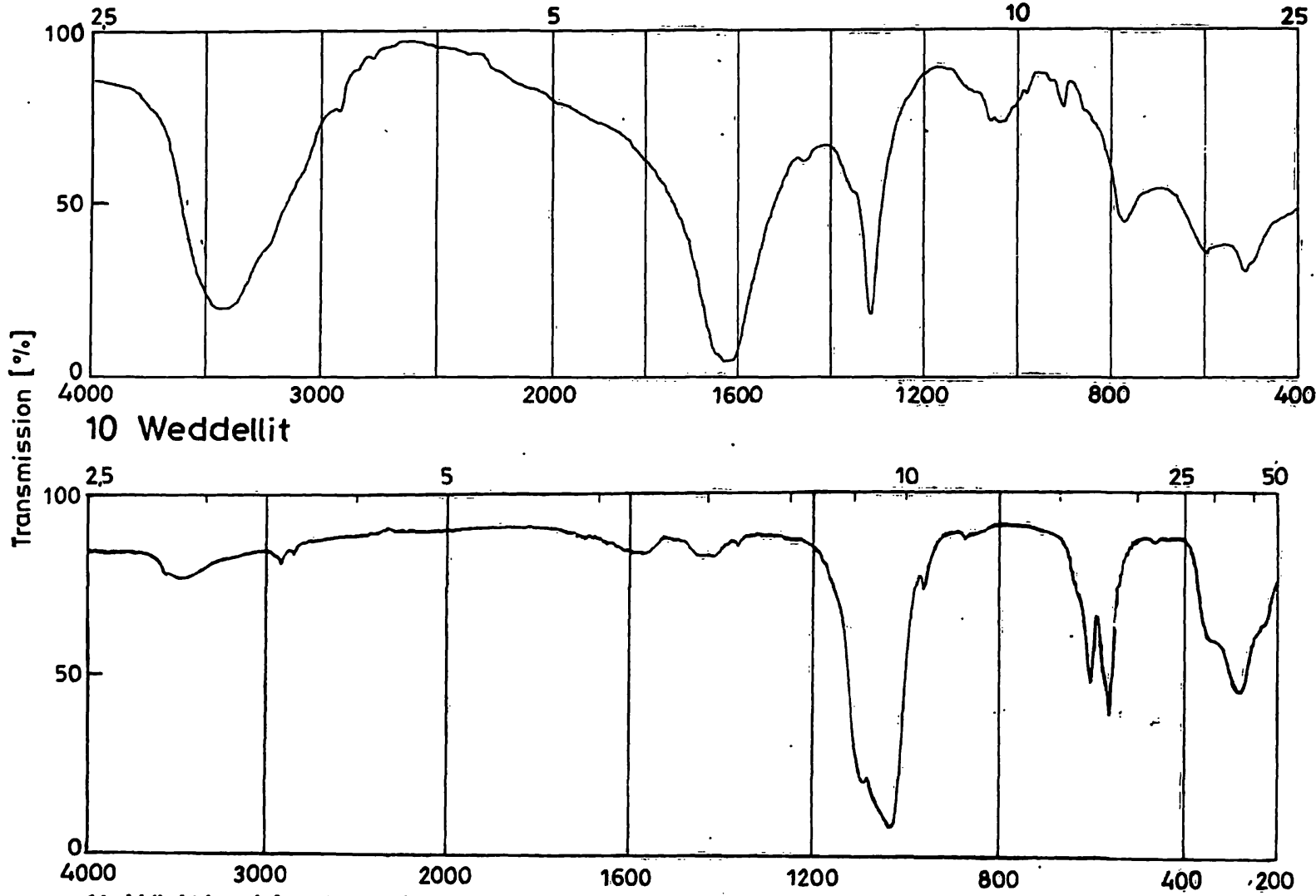

11 Whitlockit, Apatit

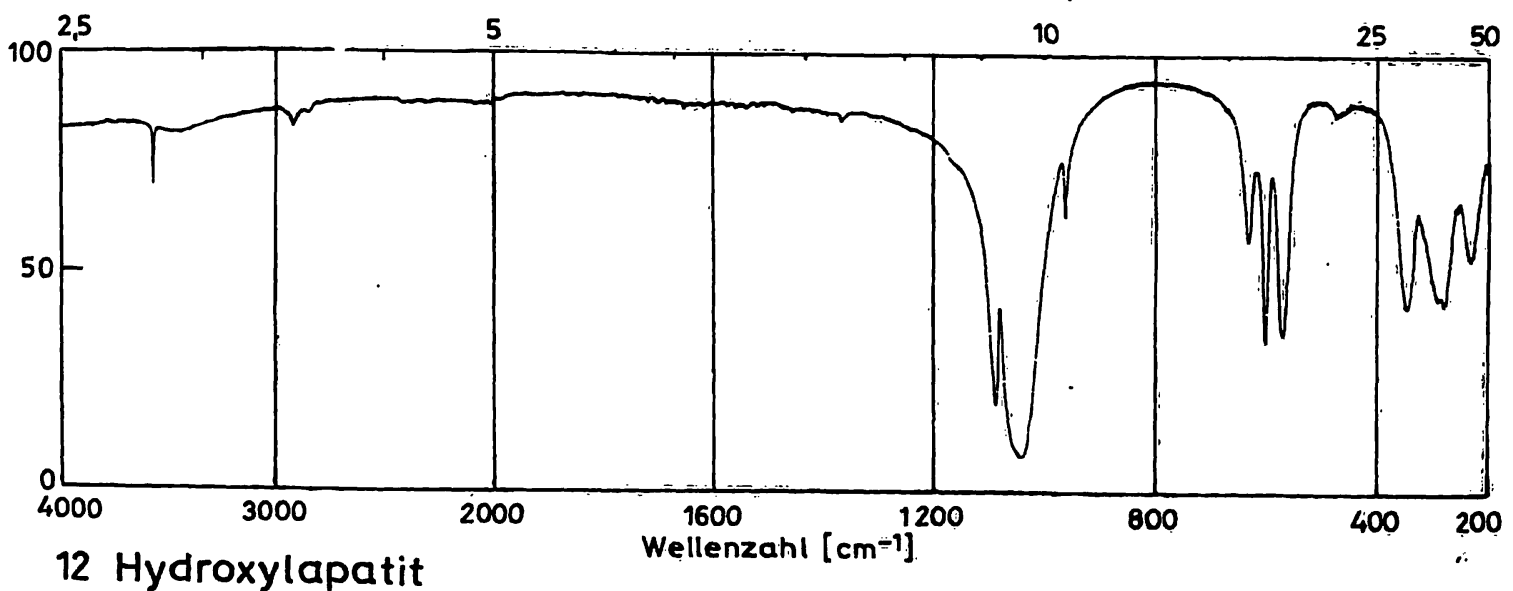

Tafel III Infrarotspektren von

9 Whewellit

11. Whitlockit, Apatit (beachte den Maßstab!)

10 Weddellit

12 Hydroxylapatit (beachte den Mäßstab!)

Spektren reiner Substànzen ạls Vergleichs-Standards zur Härnsteinanalyse 


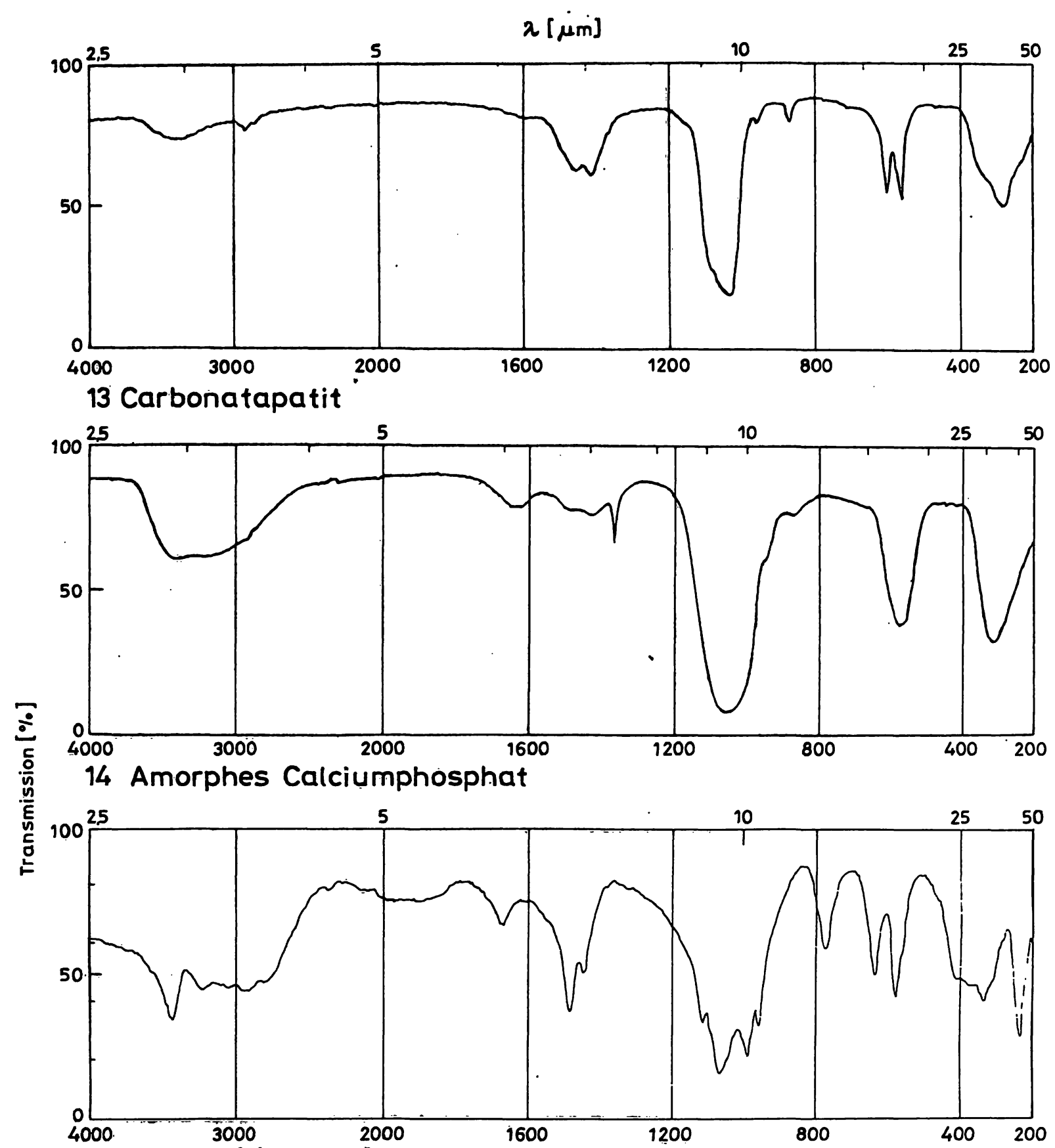

$15 \mathrm{Mg} \mathrm{NH} 4 \mathrm{PO}_{4} \cdot 1 \mathrm{H}_{2} \mathrm{O}$

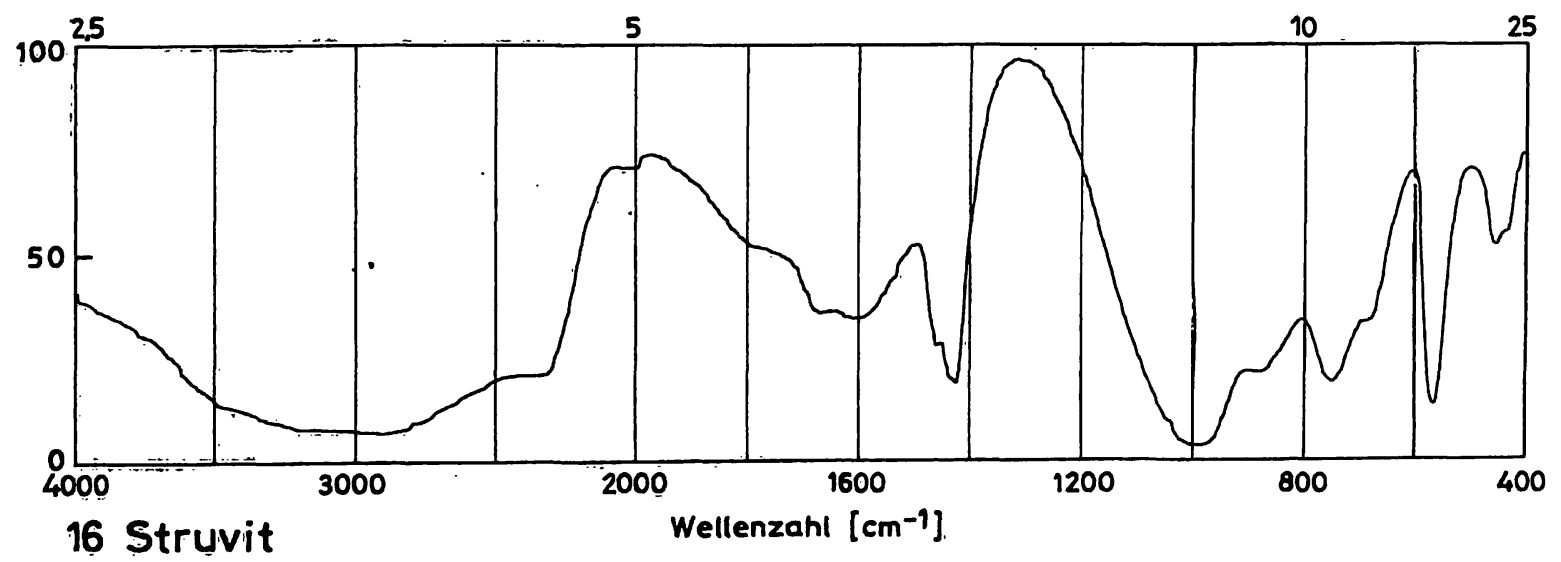

Tafel IV Infrarotspektren von

13 Carbonatapatit (beachte den Maßstab!)

14 Amorphene Calciumphosphat (beachte den Maßstab!)
15 Magnesium-ammonium-phosphat-Monohydrat (beachte den Maßstab!) 16 Struvit

Spektren reiner Substanzen als Vergleichs-Standards zur Harnsteinanalyse 


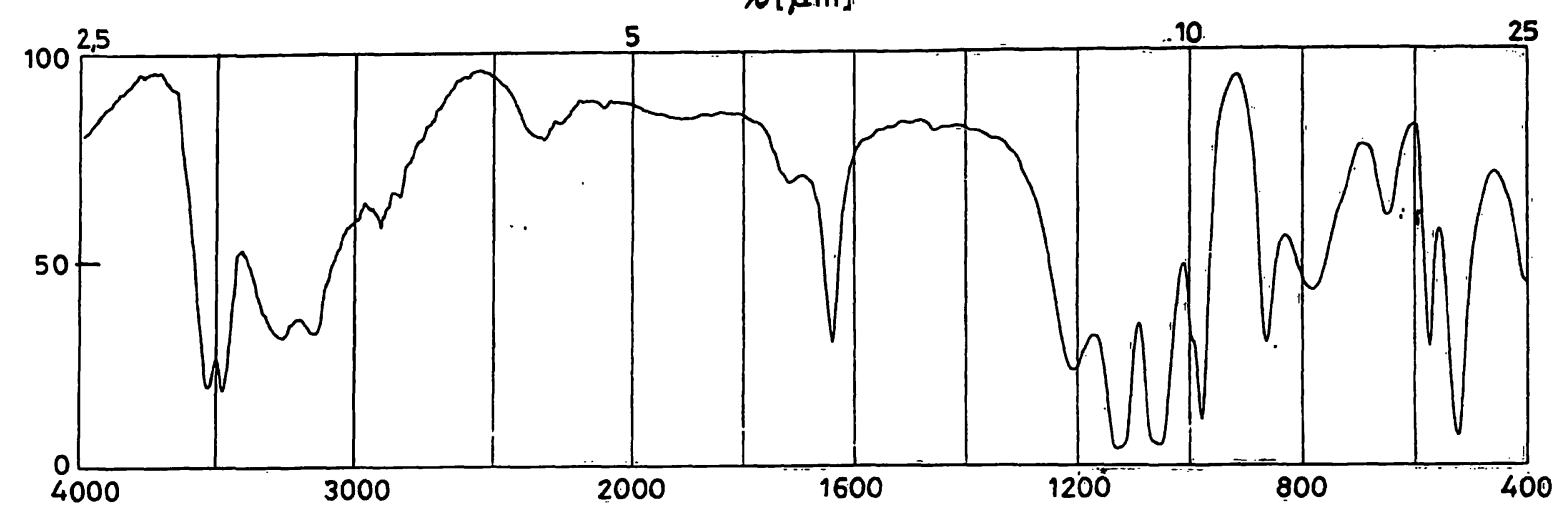

17 Brushit
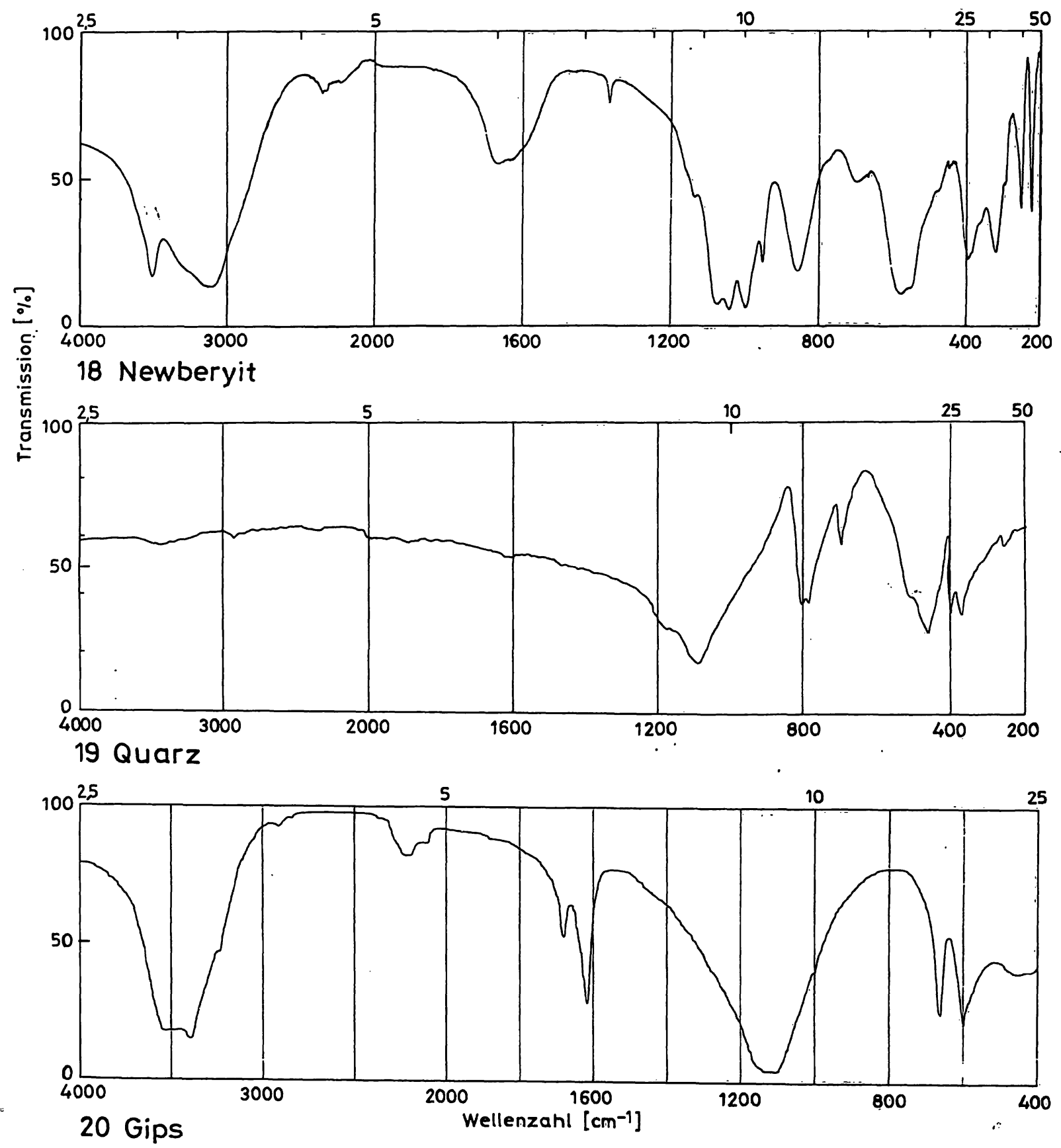

Tafel V Infrarotspektren von 17 Brushit

18 Newberyit (beachte den Maßstab!)
19 Ouarz (beachte den Maßstab!)

20 Gips

Spektren reiner Substanzen als Vergleichs-Standands zur Harnsteinanalyse 


\section{8, 19, 20 Newberyit, Quarz ( $\mathrm{SiO}_{2}$ ), Gips}

Diese Substanzen wurden bisher nur in Harnsteinen von Tieren nachgewiesen. Die IR-Spektren weichen charakteristisch von denen der Harnsteine des Menschen ab. Besonders Gips und Quarz (Kieselstein) werden gelegentlich als Artefakte von den Patienten abgegeben.

\section{Calciumcarbonat (Calcit)}

Calciumcarbonat kann in 3 Kristallsystemen, als Calcit, Aragonit und Vaterit, kristallisieren. Der Nachweis in Harnsteinen des. Menschen ist nicht gesichert. Beim positiven Carbonatnachweis liegt stets Carbonatapatit vor. In Steinen von Tieren wurde Calcit bestimmt.
Aragonit bzw. Vaterit kann in Gallensteinen und Prostatasteinen vorkommen. Die drei Modifikationen unterscheiden sich in Lage und Zahl der Hauptabsorptionen.

Calcit $\quad 708,(842), 872,1420 \mathrm{~cm}^{-1}$

Argonit $\quad 700,712,(852), 860,878,1084,1408 \mathrm{~cm}^{-1}$ Vaterit $\quad 742,(850), 878,1090,1403-1480 \mathrm{~cm}^{-1}$

\section{Cholesterin}

Cholesterin ist ein Hauptbestandteil der Gallensteine. Die Absorption bei $1051 \mathrm{~cm}^{-1}$ und die Bandengruppen zwischen $1360-1465 \mathrm{~cm}^{-1}$ sowie $2800-3000 \mathrm{~cm}^{-1}$ sind typisch.

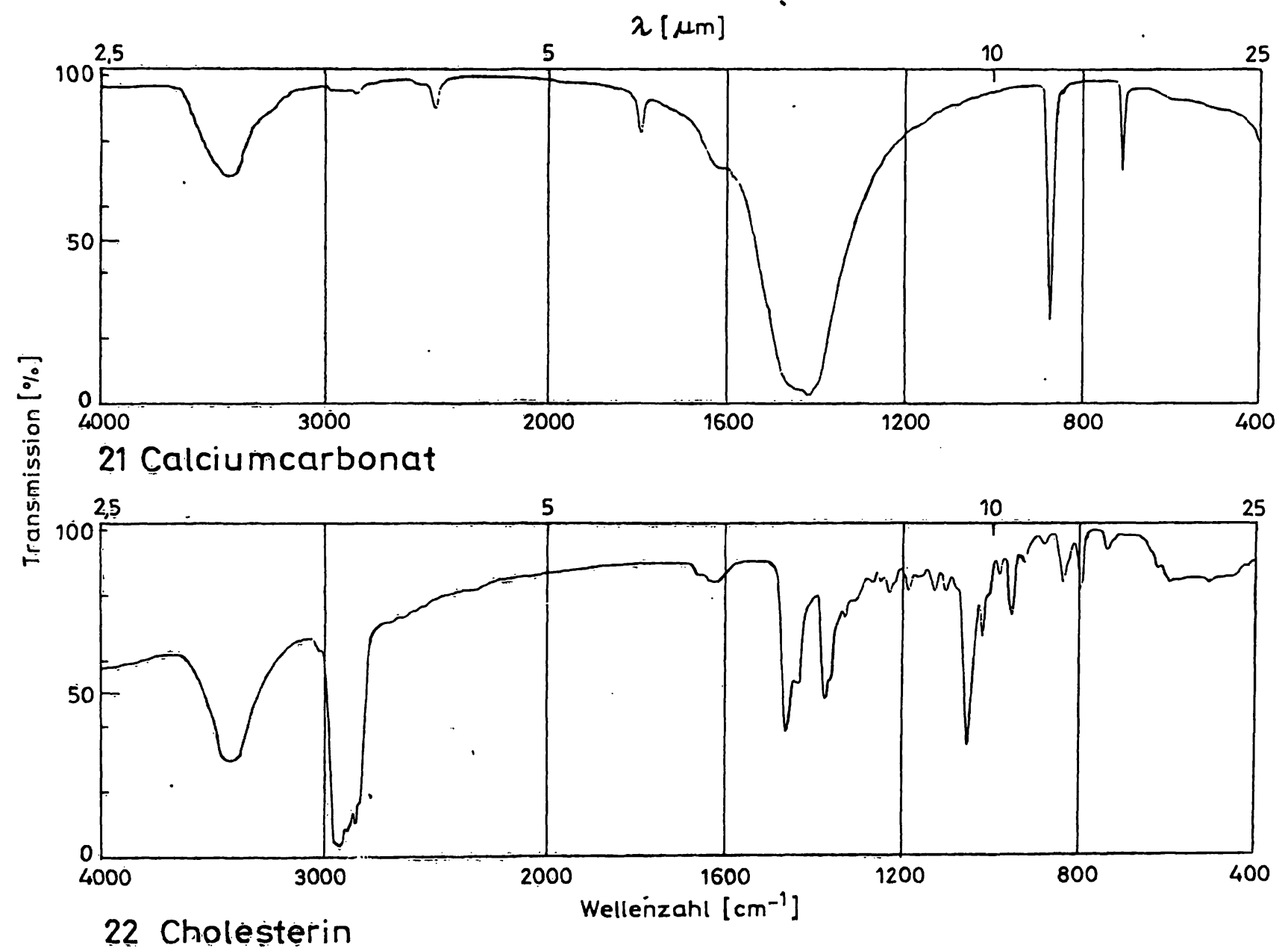

Tafel VI Infrarotspektren von

21 Calciumcarbonat

22 Cholesterin

Spektren reiner Substanzen als Vergleichs-Standards zur Harnsteinanalyse 
Auswertung zur qualitativen Analyse

Aus den vorangegangenen Erläuterungen der wichtigsten IR-Spektren für reine in Harnsteinen auftretende Verbindungen kann abgeleitet werden, daß durch Zuordnung der charakteristischen Banden eine qualitative Analyse keine Schwierigkeiten bereitet. Auch in Mehrstoffgemischen konnte in einem IRSpektrum von $4000-400 \mathrm{~cm}^{-1}$ die einzelne Verbindung im Harnstein ab Anteilen von 0,05-0,10 nachgewiesen werden. Durch eine differenzierte Präparation aus mehreren Steinabschnitten wurde die Analyse noch verbessert. Die Testspektren sollten jeweils mit dem zur Verfügung stehenden Gerät aufgenommen und mit den „Literaturspektren" verglichen werden. Durch Aufnahme von Teilspektren, bei zu geringer Auflösung oder schlechter Justierung der Geräte können die Spektren ungeeignet für eine eindeutige Auswertung werden. Für die qualitative Analyse stehen verschiedene Auswertungsverfahren zur Verfügung:

\section{Zuordnung einzelner charakteristischer Absorptionen} anhand von Vergleichsspektren,

2. Aufstellung und Vergleich von Bandentabellen,

3. Rechneràuswertung.

Ohne Datenverarbeitung hat sich für uns die Kombination der Methoden 1) und 2) für die Routineanalyse bewährt.

\section{Quantitative Analyse}

Für eine quantitative infrarotspektroskopische Harnsteinanalyse wurden die bekannten Zweistoff-

Kombinationen in Harnsteinen aus synthetischem Material hergestellt. 10-Prozent-Abstufungen sind dafür ausreichend.

Bei Verwendung eines Rechners können die Mischspektren aus den Spektren der Reinsubstanzen simuliert werden.

\section{Die Auswertung erfolgt entweder}

1. durch Einordnung in die Testspektren (Transmission oder Absorption),

2. durch manuelle oder rechnerische Auswertung von charakteristischen Banden (Transmission oder Absorption) mit Hilfe von Standardkurven.

Die angegebenen quantitativen Methoden lassen sich an den modernen IR-Geräten durch Aufnahme der Spektren im linearen Absorptionsmaßstab am besten verwirklichen. Die Auswertung wird an nicht koinzidierenden, ausgewählten Analysenbanden vorgenommen. Dabei kann die Bandenhöhe oder Bandenfläche vermessen werden.

Auf einer Minidiskette wurde eine Testbibliothek der wichtigsten in Harnsteinen enthaltenen Substanzen und einiger in der Praxis relevante Mischungen abge- speichert. Eine Bibliothekssuche einschließlich Inter' pretation benötigt etwa 20 Sekunden. Als Ergebnis wird eine Folge der ähnlichsten Substanzen ausgedruckt (Tab. 2). Jeder Substanz sind zwei Ziffern zwischen 0 und 9 vorangestellt, welche die U'bereinstimmung mit dem aufgenommenen Spektren zeigẹn, wobèi 0 keine und 9 maximale Übereinstimmung bedeutet.

Tab. 2. Rechnerauswertung zur qualitativen infrarotspektroskopischen Harnsteinänalyse. Ausdruck der Reihenfolge ähnlichster Substanzen.

HEAREST FIFTEEN SPECTRA FROM LIBRARY : HEHA PEAKM 05

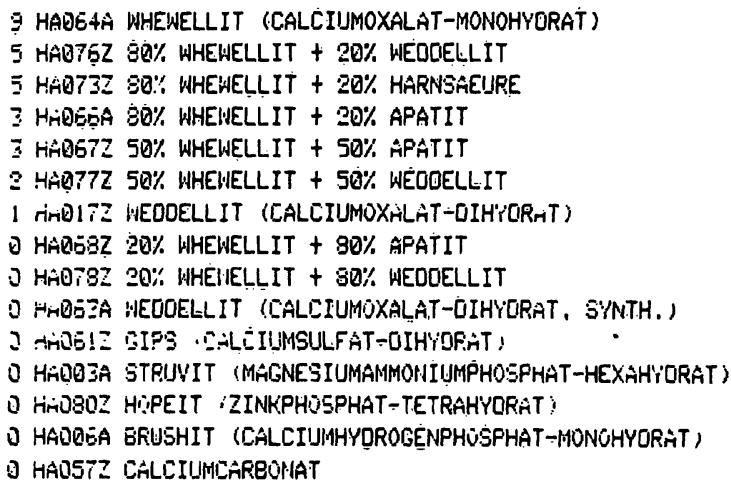

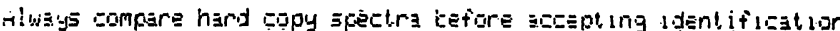

Die erste Ziffer bezieht sich auf die Strukturübereinstimmung, die zweite auf die Ubereinstimmung der Bandenlagen. Zusätzlich steht vor jeder ausgedruckten Verbindung ein Hinweis auf die Nummer des Spektrums in einer Spektrensammlung, waš einen raschen Spektrenvergleich zur endgültigen Identifizierung ermöglicht. Auf diese Bestätigung des Ergebnissses durch visuellen Vergleich sollte auf keinen Fall verzichtet werden.

Für Gemische steht ein speziell entwickeiter Suchalgorithmus (MIXSEARCH) zur Verfügung. Abbildung 1 zeigt ein normiertes Harnsteinspektrum, wobei es

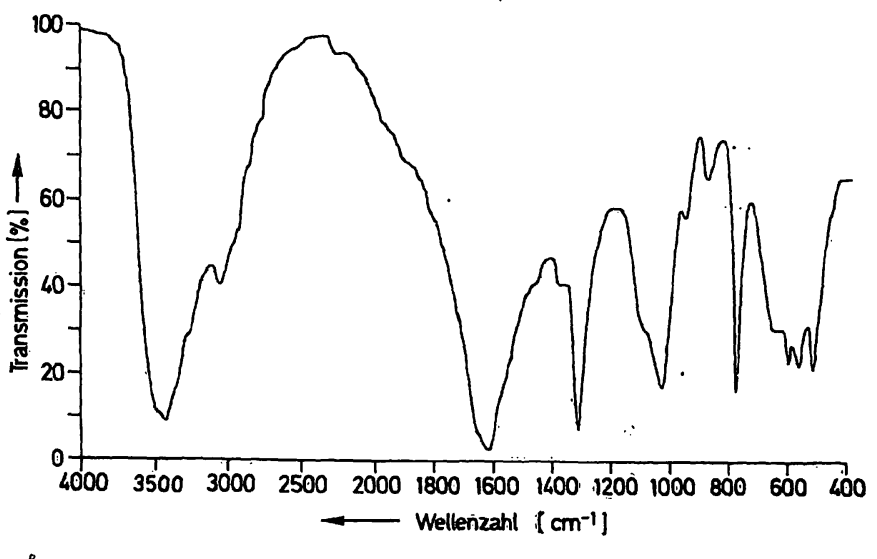

Abb. 1. Normiertes IR-Spektrum eines Ca-Oxalat-/ApatitMischsteines. 
sich offensichtlich um einen Apatit/Ca-Oxalat-Mischstein handelt. Das Ergebnis der automatischen Bibliothekssuche zeigt Tabelle 3. Die besten Ubereinstimmungen weisen Whewellit (9-3), Carbonatapatit $(8-3)$ und ein Gemisch aus $80 \%$ Whewellit und 20\% Apatit (9-4) auf. Dies ist ein eindeutiger Hinweis darauf, daß es sich bei der vorliegenden Probe um ein Gemisch handelt. Die Hauptkomponenten werden vom SEARCH-Programm richtig erkannt.

Tab. 3. Rechnerauswertung zur quantitativen infrarotspektroskopischen Harnsteinanalyse.

IIEAREST FIFTEEN SFECTRA FFOM LIBRARY : H44 MIXS 05

9-4 HAO66A 80\% WHEWELLIT + 20\% APATIT

Q-3 HAOSTH WHEWELLIT (CALCIUAOXALAT-MONOHYORAT)

9-1 HAQG7Z 50\% WHEINLLIT + 50\% APATIT

9-D HAOT2Z 20\% STRUVIT + 80\% APATIT

Q-0 HAOOSA BRUSHIT (CALCIUMHYOROGENPHOSPHAT-MONOHYORAT)

9-0 HAQ38A AMINOHIIIIURAT

Q-0 HADIGZ RIIIDERALBUHIII

O-3 HAD4OZ LAFBOIIATAPATIT

3-1 HAO6I: GIPS (CALCIUMSILFAT-OIHVIORAT)

S-1 HAQTEL 80\% WHEWELLIT + 20\% WEOUELLIT

8-1 HAOT3Z $80 \%$ WHEWELLIT + $20 \%$ HAFNSAEURE

S-1 HAO68: $20 \%$ WHEMELLIT + 80\% APATIT

8-0 HA05TZ CALIIUMCARBOHAT

8-6 HABSIZ VATEFIT (CALCIUMCARBINAT)

8-0 HAOTBZ $20 \%$ HHEMELLIT + $80 \%$ WEODELLIT

Always compare hard copy spectra before accepting identification

Die Vollständigkeit der Bibliothek würde voraussetzen, daß eine große Zahl von der in der Praxis vorkommenden Mischungen synthetisiert und aufgenommen würde, was zwar in Bezug auf die Speicherkapazität des Systems unproblematisch ist, jedoch einen sehr hohen präparativen Arbeitsaufwand erfordert. Da dieser jedoch nur einmal aufzubringen ist, erscheint dies lohnenswert.

Rechnerunterstützte Auswertung der IR-Spektren von Harnsteinen

Der Zeitaufwand für ein IR-Spektrum einschließlich Probenpräparation beträgt etwa 10-15 Minuten. Hieran schließt sich jedoch noch die Auswertung an, und dies ist der kritischste Schritt im gesamten Analysenablauf. Um den Informationsgehalt eines IR-Spektrums schnell und richtig auszuwerten, bedarf es einiger Erfahrung. Im allgemeinen wird das Spektrum an Hand charakteristischer Banden zunächst einer bestimmten Verbindungsklasse zugeordnet und anschließend zur genauen Identifizierùng mit den in Frage kommenden Spektren einer Spektrensammlung verglichen. Hierbei ist zu beachten, daß Harnsteine aus einem Gemisch mehrerer Substanzen bestehen können, was die Auswertung weiter kompliziert.
Sowohl für die Aufgabe der Spektreninterpretation wie auch für die Bibliothekssuche bietet sich die Unterstützung durch einen Computer an. Es kann hierfür ein in der Praxis der analytischen Chemie bereits bewährtes Identifizierungsprogramm mit dem Namen SEARCH, das auf einem on-line mit dem IR-Gerät gekoppelten Tischrechner („IR-Datenstation") läuft (6), verwendet werden. Das Spektrum wurde dafür mit einer konstanten Schrittweite von $1 \mathrm{~cm}^{-1}$ digitalisiert, rechnerisch geglättet und so normiert, daß die maximale Transmission $100 \%$ und die minimale $3,16 \%$ (0-1,5 Absorptionseinheiten) beträgt. Die normierte Darstellung ist vorteilhaft für Vergleichszwecke, da die Bandenintensitäten unabhängig von der eingewogenen Substanzmenge werden, vorausgesetzt, daß keine Transmissionswerte. unter $3,16 \%$ auftreten.

Um letzteres zu vermeiden, wurden Einwaagen von deutlich unter $1 \mathrm{mg}$ Substanz pro $300 \mathrm{mg}$ Kaliumbromid verwendet. Abbildung 2 zeigt das normierte

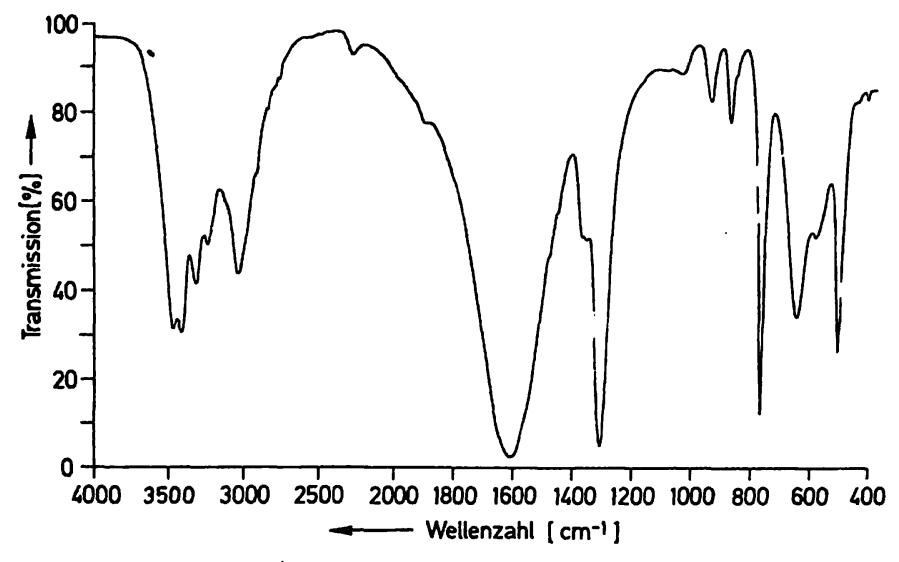

Abb. 2. Normiertes IR-Spektrum von Whewellit.

Spektrum des Whewellits. Hiervon wird automatisch eine Bandentabelle erstellt, die für jede Absorptionsbande die Wellenzahl und den Transmissionswert enthält (Tab. 4). Diese Bandentabelle wird vom SEARCH-Programm ausgewertet. Hierbei erfolgt im ersten Schritt zunächst eine Interpretation mit Ausgabe der möglichen Struktureinheiten (PSU's = Possible Structural Units, Tab. 5). Eine PSU ist hierbei definiert als ein Molekülbaustein, eine funktionelle Gruppe oder eine Kombination von funktionellen Gruppen, die an Hand des IR-Spektrums durch

Tab. 4. Banden $\left(4000-400\left[\mathrm{~cm}^{-1}\right]\right)$ und Transmissionswerte (3,16-100,00 [\%] vom IR-Spektrum des Whewellits.

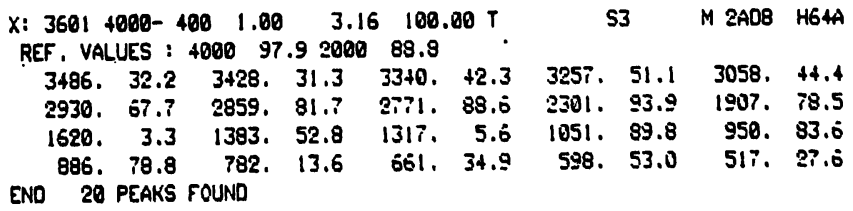


eine bestimmte Anordnung charakteristischer Banden erkannt werden können. Nach der Interpretation wird eine automatische Bibliothekssuche durchgeführt. In der Bibliothek sind für jede Substanz die Bandenfrequenzen und die PSU's gespeichert. Bei der Suche werden wahlweise nur die Bandenlagen oder die Bandenlagen und die Molekülstruktur verglichen.

Tab. 5. Zuordnung möglicher Struktureinheiten für das IRSpektrum des Whe wellits.

POSSIBLE STRUICTURAL UNITS : HEHA

4704 CARBOXYLIC ACIO SALT - FOSSIBLY OXALATE

470 CARBOXYLIC ALIO SALT - SHORT CHAIN COMPOIJNO

PSU: above may be sub.ject to interference. Consult, Manual

\section{Diskussion}

Die spektroskopischen Methoden sind wichtige Untersuchungstechniken zur Bestimmung der Struktur von Molekülen. Neben der Geometrie eines Moleküls werden dabei die Elektronenstruktur und damit die Bindungsverhältnisse charakterisiert. In Abhängigkeit von der eingestrahlten Energie bzw. Wellenlänge der Strahlung werden Elektronen-, Schwingungs- und Rotationszustände der Moleküle angeregt. Unter den Bedingungen der hier interessierenden IR-Spektroskopie entsprachen die Absorptionen den Veränderungen der Schwingungszustände im Molekül. Im infraroten Spektralbereich (2,5-50 $\mu \mathrm{m}$ bzw. $\left.4000-200 \mathrm{~cm}^{-1}\right)$ können in Molekülen und Kristallen Schwingungen angeregt werden, die eine Änderung des Dipolmomentes des schwingenden Systems bewirken. Durch diese Wechselwirkung der infraroten Strahlung mit der zu untersuchenden Substanz treten in Abhängigkeit von den Schwingungsmöglichkeiten der Moleküle bei bestimmten Wellenlängen ganz charakteristische Schwächungen der eingestrahlten Energie auf. Bestimmte Absorptionen sind charakteristisch für einzelne Atomgruppen in Molekülen. Auf dieser Basis ist auch die eindeutige Unterscheidung der in Harnsteinen auftretenden Substanzen möglich. In Tabelle 6 sind die Absorptionsbereiche für einige Atomgruppen aufgeführt, die zur Identifizierung von in Harnsteinen vorkommenden Substanzen eine Rolle spielen.

In Abhängigkeit von den benachbarten Gruppen und der Gesamtstruktur des Moleküls haben in den einzelnen Verbindungen die Absorptionen eine genaue Lage im Infrarot-Spektrum.

Absorptionen im infraroten Spektralbereich sind unabhängig vom Kristallisationsgrad der Probe. Dadurch ist es möglich, auch schlecht kristallisierte-Substanzen häufig sind die Calciumphosphate in Harnsteinen pseudo- amorph - und auch amorphe Verbindungen, wie z.B. Eiweiße (organische Matrix), nachzuweisen.

Die Abhängigkeit der Infrarotspektren von den Schwingungsmöglichkeiten des Moleküls kann bei geringen Unterschieden im Molekuilbau zu Schwięrigkeiten der Identifizierung einzelner Substanzen in Gemischen führen. Bei den in Harnsteinen enthaltenen Substanzen unterscheiden sich Whewellit und Weddellit sowie Harnsäure und Harnsäure-Dihydrat nự durch den Kristallwassergehalt. Für Whewellit und Weddellit erlauben die IR-Spektren auch in Gemischen eine sichere Differenzierung. Dagegen sind die Absorptionen von Harnsäure und Harnsäure-Dihydrat sehr ähnlich, so daß reinè Substanzen zwar noch sehr gut ermittelt werden können, aber Harnsäure-Dihydrat als Mischpartner häufig nicht von Harnsäure unterschieden werden kann.

Für eine genaue qualitative und quantitative Harnsteinanalyse ist die Anwendung eines Infrarotspektrometers mit dem Wellenzahlbereich $4000-400 \mathrm{~cm}^{-1}$ erforderlich. Heute stehen dispersive Gittergeräte und Fourier-IRGeräte zur Verfügung, wobei letżtere im Anschaffungs: preis jedoch relativ hoch liegen. Die Investitionskosten für ein geeignietes dispersives Gerät mit Zubehör liegèn bei 30000-40000 DM. Die Ausrüstung mit einer Datenstation ist nư für Steinanalyssezentren sinnvoll. Es müssen dafür ẑum jetzigen Żeitpunkt (1982) zusätzlich etwa 40000 DM aufgewendet werden.

Nach den mit der Testbibliothek gesammelten Erfahrungen halten wir es für möglich, dảß sich durch systematischen Ausbau diesèr Bibliothek ein generell ein: setzbares System für die automatische qualitative und halbquantitative Harnsteinanalyse entwickeln läßt. Soll darüber hinaus eine genaue quañtitative Analyse erfolgen, muß dies durch direkte Auswertung der $\mathrm{Ab}$ sorptionen von substanzspezifischen Schlüsselbanden erfolgen, wie in einer früheren Arbeit beschrieben (7). Auch hierbei können rechnerunterstütżte Verfähren die Genauigkeit und Geschwindigkeit der Auswertung wesentlich verbessern, was wir an anderer Stelle zeigen wollen.

Tab. 6. Charakteristische Absorptionsbereiche von Atomgruppen in Harnsteinen vorkommender Substanzen.

\begin{tabular}{|c|c|c|}
\hline \multirow[t]{2}{*}{ Atomgruppe } & \multicolumn{2}{|c|}{$\begin{array}{l}\text { Charakteristische Schwingung } \\
\left(\mathrm{cm}^{-1}\right)\end{array}$} \\
\hline & Valenzschwingung & $\begin{array}{l}\text { Deformations- } \\
\text { schwingung }\end{array}$ \\
\hline $\begin{array}{l}\mathrm{C}-\mathrm{N} \\
\mathrm{C}-\mathrm{O}\end{array}$ & $\begin{array}{l}2500-3000 \\
1600-1800\end{array}$ & $1300-1500$ \\
\hline O-H & $3100-3600$ & $\begin{array}{l}1100-1400 \\
1650\left(\mathrm{H}_{2} \mathrm{O}\right)\end{array}$ \\
\hline $\begin{array}{l}\mathrm{N}-\mathrm{H} \\
\mathrm{NH}^{4+} \\
\mathrm{PO}_{4}^{3-} \\
\mathrm{CO}_{3}^{3-}\end{array}$ & $\begin{array}{l}3200-3500 \\
3030-3300 \\
1000-1100 \\
1350-1450\end{array}$ & $\begin{array}{r}1550-1650 \\
1390-1430 \\
550-650 \\
850-900\end{array}$ \\
\hline
\end{tabular}




\section{Literatur}

1. Hesse, A., Röhle, G. \& Voigt, U. (1982) Fortschr. Urol. Nephrol. 17, 306-310.

2. Hesse, A., Hicking, W. \& Vahlensieck, W. (1981) GIT Labor-Medizin 4, 19-27.

3. Gebhardt, M. (1979) Harnsteinanalyse mittels Röntgendiffraktion in: Urolithiasis 1 (Vahlensieck, W., ed.) Springer Berlin, Heidelberg, New York, S. 77-121.
4. Beischer, D. E. (1955) J. Urol. (Baltimore) 73, 653-659.

5. Hesse, A., Schneider, W.-J. \& Hienzsch, E. (1972) Dtsch. Med. Wochenschr. 97, 1694-1701.

6. Molt, K. (1980) Chemie-Technik 9, 195-200.

7. Hesse, A., Schrumpf, G. \& Schilling, I. (1974) Zschr. Urol. 67, 367-376.
Dr. A. Hesse

Urologische Univ. Klinik Sigmund-Freud-Str. 25

D-5300 Bonn 
。 